\title{
Acute pancreatitis as the initial presentation in systemic lupus erythematosus
}

\author{
(1) Samarth Virmani, (1) Shubha Seshadri, (1) Sharath Madhyastha P., (1) Akhya Sharma, (1) Charan Thej Reddy
}

Manipal Academy of Higher Education, Kasturba Medical College, Clinic of Medicine, Manipal, India

Date submitted:

05.07.2020

Date accepted:

31.08.2020

Online publication date:

15.06.2021

\section{Corresponding Author:}

Sharath Madhyastha P. Assoc. Prof., M.D, Manipal Academy of Higher Education, Kasturba Medical College, Clinic of Medicine, Manipal, India

ORCID:

orcid.org/0000-0003-2821-3473

Keywords: Acute pancreatitis, systemic lupus erythematosus, steroids

\begin{abstract}
Systemic lupus erythematosus (SLE) is an autoimmune connective tissue disorder involving multiple organ systems with varying manifestations. Acute pancreatitis as an initial presenting feature of SLE is extremely rare. A 21-year-old female patient presented with fever and severe abdominal pain for the past 20 days. Her history was unremarkable except for an abortion two months ago. She was diagnosed with both acute pancreatitis and SLE following a thorough evaluation.
\end{abstract}

\section{Introduction}

Systemic lupus erythematosus (SLE) is an autoimmune disease that affects almost every organ of the body. Gastrointestinal tract involvement is seen in almost $50 \%$ of the cases. Acute pancreatitis as a complication of SLE is rare and mostly a flare-up in patients already having a preceding diagnosis of SLE. The frequency of SLE-associated pancreatitis is estimated between 0.2 and $8.2 \%$. Such patients are known to have a higher disease activity index score as well as increased mortality. Here, we present a young patient who presented with acute pancreatitis and was diagnosed with SLE during her evaluation.

\section{Case Presentation}

A 21-year-old woman free of any comorbidities was admitted to the emergency room with abdominal pain and fever in the past 20 days. The pain was severe, non-radiating, and not associated with any aggravating or relieving factors. The grade of fever was low, which also showed an intermittent pattern. She also complained of vomiting following food intake in the last two weeks, which was non-bilious and non-bloody but contained food particles. She did not report regular alcohol intake and reported an abortion two months before her current complaints.

On physical examination, the patient looked distressed. Arterial blood pressure was 90/60 mmHg, pulse was 110 beats/ minute, and oxygen saturation was $99 \%$ in the room. She was afebrile. There was severe tenderness in the epigastric area, with no palpable organomegaly. Bowel sounds were normal.

Routine blood tests revealed pancytopenia (hemoglobin: $10.9 \mathrm{~g} / \mathrm{dL}$, leucocyte count: 2260/ $\mathrm{mm}^{3}$, and platelet count: $71,000 / \mathrm{mm}^{3}$ ). Liver function tests showed hypoalbuminemia (serum albumin: $2.96 \mathrm{~g} / \mathrm{dL}$ ) with normal liver enzymes and bilirubin levels. Serum amylase and lipase levels were elevated (1270 U/L and $2732 \mathrm{U} / \mathrm{L}$, respectively). Abdominal computed tomography (CT) revealed acute interstitial edematous pancreatitis with moderate ascites and bilateral mild pleural 
effusion (Figure 1). Serum triglyceride and calcium levels were normal. Microlithiasis and pancreatic divisum were excluded by endoscopic ultrasonography. Due to the history of abortion, an autoimmune disease panel was studied, which showed antineutrophil antibody was strongly positive with anti-dsDNA titer $>800 \mathrm{IU} / \mathrm{mL}$.

Serum creatinine and glomerular filtration rate were normal $(0.8 \mathrm{mg} / \mathrm{dL}$ and $90 \mathrm{~mL} / \mathrm{min}$, respectively). Dipstick urine examination revealed $3+$ proteinuria with no casts or crystals on microscopy. 24-hour urine protein level was $390 \mathrm{mg} / 24$ hours.

The patient underwent kidney biopsy, which revealed diffuse global proliferative glomerulonephritis ISN class $4 \mathrm{~A}$ with thrombotic microangiopathy (activity score of 10/24 and chronicity score of 0/12). Serum C3 and C4 (10 U/mL and $3 \mathrm{U} /$ $\mathrm{mL}$, respectively) were also decreased.

The patient was started treatment with acute pancreatitis (AP) protocol. Following the diagnosis of SLE-induced pancreatitis, intravenous (IV) steroid treatment was added (dexamethasone $4 \mathrm{mg} \mathrm{Q8H}$ ). Once her condition improved, she was switched to oral prednisolone (1 mg/kg body weight) and mycophenolate mofetil ( $1 \mathrm{~g}$ twice daily) due to lupus nephritis. The patient's condition gradually improved after the initiation of steroid therapy in around five days, and serum amylase and lipase also started to decrease. The patient is currently on regular follow-up without symptoms.

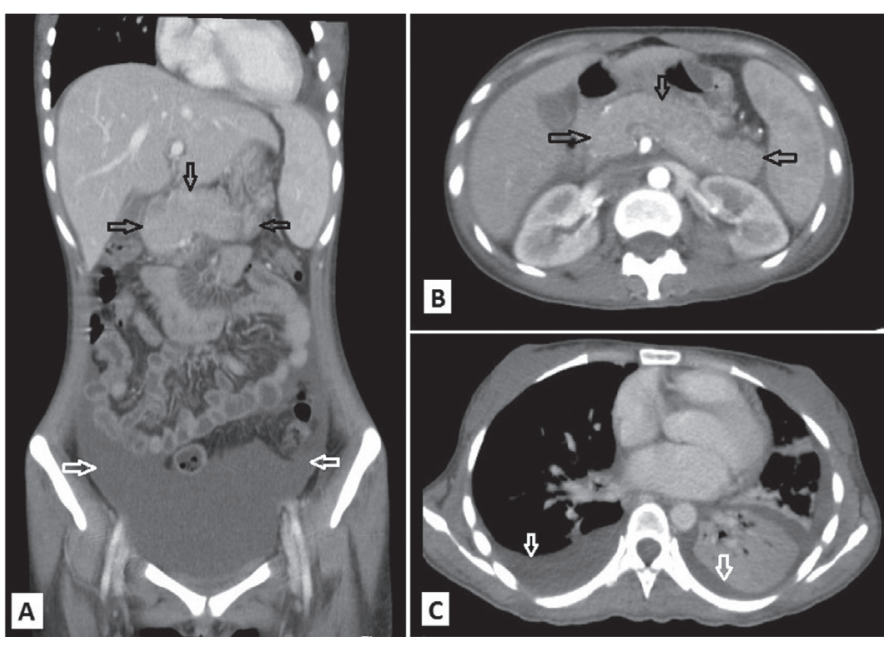

Figure 1. Computed tomography of abdomen (A: coronal, $B, C$ : axial views): Pancreas appears bulky in size (A, B: black arrows) with minimal peripancreatic fluid collection. Mild to moderate ascites (A: white arrows) and bilateral mild pleural effusion (C: white arrows)

\section{Discussion}

SLE is a chronic autoimmune condition that can potentially affect various organ systems. The incidence of gastrointestinal (GI) manifestations is commonly underestimated due to the lack of significant abdominal symptoms in most patients (1).
GI involvement and symptoms are seen in about $19.2-50 \%$ of patients with SLE $(2,3)$.

Lupus-associated acute pancreatitis is one of the GI manifestations in SLE. SLE-associated acute pancreatitis has been associated with very high SLE Disease Activity Index Scores and extremely high mortality rates of approximately $10.3-68.4 \%(4,5)$.

The pathogenesis of SLE-associated pancreatitis is complex. Vascular damage (which includes vasculitis, intimal thickening, immune complex microunits, as well as occlusion of arteries and arterioles), excess production of autoantibodies, and abnormal cellular immune response are among the hypothesized factors (2). The major risk factors associated with SLE-associated pancreatitis are hypertriglyceridemia, psychosis, recent viral infection like cytomegalovirus, and drug toxicity $(6,7)$. As mentioned above, our patient had no such significant risk factors for AP.

Patients with SLE-associated pancreatitis can have a wide range of presenting symptoms from asymptomatic (an elevation of pancreatic enzymes being the only abnormality) to acute (severe and/or fulminant) or chronic (self-limiting) features (8). Abdominal pain is the most common documented symptom, seen in about $90 \%$ of patients, along with nausea and vomiting in $75 \%$ and fever in $50 \%$ of the patients $(1,4,7)$.

The diagnosis of acute pancreatitis is solely dependent on the presenting symptoms, physical examination, and basic diagnostic workup (e.g., pancreatic enzymes), supported by appropriate imaging. Elevation of serum amylase and lipase levels is the most common laboratory abnormality in lupus pancreatitis, although normal levels are not uncommon $(1,7)$. In addition, other significant biochemical abnormalities noted were hypoalbuminemia $(78 \%)$, liver function test abnormalities $(65 \%)$, and elevated serum creatinine $(44 \%)(1,4)$. Leukocytosis is relatively uncommon, occurring in only about $15 \%$ of the patients, whereas anemia, leukopenia, and thrombocytopenia have been seen in $81 \%, 59 \%$, and $48 \%$ of cases in the literature, respectively (4). Our patient had elevated serum amylase and lipase levels with hypoalbuminemia, anemia, leucopenia, thrombocytopenia, and proteinuria.

Multiple factors are associated with increased mortality risk in patients with lupus-associated pancreatitis. Acute renal failure and thrombocytopenia may complicate a severe disease activity $(4,7,9)$. The concern regarding steroid treatment is minimal for treatment of SLE in acute pancreatitis patients despite the chance of a toxic effect due to steroids. Previous studies have suggested steroid administration during an acute event of SLE pancreatitis $(4,5,10)$. Our patient received IV steroids during hospitalization, followed by oral steroids at discharge, and was later started on mycophenolate mofetil to manage lupus nephritis. 


\section{Conclusion}

Although rare, SLE-associated pancreatitis should be suspected in a patient with acute abdominal pain after excluding the common causative factors. Identification of SLE-associated symptoms may be helpful during evaluation. Lupus-induced acute pancreatitis can gain benefit from routine treatment of SLE.

\section{Acknowledgments}

We are grateful to the department of radiology for providing contrast enhanced CT abdomen images.

\section{Ethics}

Informed Consent: Consent form was filled out by all participants.

Peer-review: Internally peer-reviewed.

\section{Authorship Contributions}

Surgical and Medical Practices: S.S., Concept: S.S., S.M.P., Design: S.M.P., Data Collection or Processing: C.T.R., Analysis or Interpretation: S.M.P., C.T.R., Literature Search: S.V., A.S., Writing: S.V., A.S.

Conflict of Interest: No conflict of interest was declared by the authors.

Financial Disclosure: The authors declared that this study received no financial support.

\section{References}

1. Tian XP, Zhang X. Gastrointestinal involvement in systemic lupus erythematosus: Insight into pathogenesis, diagnosis and treatment. World J Gastroenterol. 2010;16:2971-2977.
2. Noia JL, García FM, Ríos SS, Iglesias García J, Domínguez Muñoz JE. Pancreatitis and systemic lupus erythematosus. Revista Espanola de Enfermedades Digestivas. 2009;101:571-579.

3. Wang F, Wang NS, Zhao BH, Tang LQ. Acute pancreatitis as an initial symptom of systemic lupus erythematosus: a case report and review of the literature. World J Gastroenterol. 2005;11:4766-4768.

4. Breuer GS, Baer A, Dahan D, Nesher G.Lupus-associated pancreatitis. Autoimmun Rev. 2006;5:314-318.

5. Nesher G, Breuer GS, Temprano K. Lupus-associated pancreatitis. Semin Arthritis Rheum. 2006;35:260-267.

6. Carducci M, Calcaterra R, Mussi A, Franco G, Morrone A. Acute pancreatitis as initial manifestation of systemic lupus erythematosus and subacute cutaneous lupus erythematosus: report of two cases. Lupus. 2008;17:695697.

7. Makol A, Petri M. Pancreatitis in systemic lupus erythematosus: frequency and associated factors-a review of the Hopkins Lupus Cohort. J Rheumatol. 2010;37:341345.

8. Jia Y, Ortiz A, Mccallum R, Salameh H, Serrato P. Acute pancreatitis as the initial presentation of systematic lupus erythematosus. Case Rep Gastrointest Med. 2014;2014:571493.

9. Wang $Q$, Shen $M$, Leng $X$, Zeng $X$, Zhang F, Qian J. Prevalence, severity, and clinical features of acute and chronic pancreatitis in patients with systemic lupus erythematosus. Rheumatol Int. 2016;36:1413-1419.

10. Ramanan AV, Thimmarayappa AD, Baildam EM. Acute lethal pancreatitis in childhood systemic lupus erythematosus. Rheumatology. 2002;41:467-469. 